\title{
Capacidade de idosos da comunidade para desenvolver Atividades de Vida Diária e Atividades Instrumentais de Vida Diária
}

\author{
Elder's community capacity to develop Daily Life Activities and Daily Instrumental \\ Life Activities \\ Capacidad de los ancianos de una da comunidad en desenvlover Actividades de Vida Diaria y \\ Actividades Instrumentales de Vida Diaria \\ Efraim Carlos Costa ${ }^{1}$, Adélia Yaeko Kyosen Nakatani $^{2}$, Maria \\ Márcia Bachion²
}

\begin{abstract}
RESUMO
Objetivos: Identificar algumas características sociais e demográficas de idosos de uma comunidade em Goiânia (GO); avaliar a capacidade dos mesmos para as atividades de vida diária (AVD) e atividades instrumentais de vida (AIVD). Métodos: Estudo transversal, envolvendo 95 idosos da comunidade de uma área de abragência do Programa Saúde da Família, em Goiânia. Foram realizadas: entrevista e avaliação de AVD e AIVD. Resultados: Participaram 95 idosos, com predomínio da faixa etária de 60 a 69 anos (60\%), sexo feminino (60\%), estado civil casado (49,5\%), religião católica $(54,7 \%)$ renda menor ou igual a 1 salário mínimo $(45,3 \%)$ e moradia própria (89,5\%). Foram identificados independência máxima nas AVD (57,9\%) e comprometimento nas AIVD (72,6\%). Conclusão: Os participantes apresentaram, predominantemente, independência para as atividades no domicílio e dependência para as atividades fora dele.
\end{abstract}

Descritores: Saúde do idoso; Cuidados primários de saúde; Enfermagem geriátrica; Programa saúde da família

\begin{abstract}
Objectives: To identify social and demographic characteristics of elderly people and to measure their capability to perform activities of daily living (ADLs) and instrumental activities of dalily living (IADLs). Methods: This was transversal study, involving 95 elders from the Family Health's Program at Goiânia, Brazil. Data were colleted thought interviews and evaluations of the elderly capability to perform ADLs and IADLs. Results: Participants had an age between 60 and 69 years old $(60,0 \%)$, were women $(60,0 \%)$, married $(49,5 \%)$, Catholic $(54,7 \%)$, home owners and had income bleow or equal to a basic minimum wage $(89,5 \%)$. Participants had maximal independence in the ADLS $(57.9 \%)$ and IADLs (72.6\%). Conclusion: Most of the participants were cpable to perform indendently ADLs and IADLs.
\end{abstract}

Keywords: Aging Health; Primary Health Care; Geriatric nursing; Family Health Program.

\section{RESUMEN}

Objetivos: Identificar algunas características sociales y demográficas de ancianos de una comunidad, en Goiânia (GO); evaluar la capacidad de los mismos para actividades de la vida diaria (AVD) y actividades instrumentales de vida (AIVD). Métodos: Estudio transversal que involucró a 95 ancianos de la comunidad de uan área de competencia del Programa Salud de la Familia, en Gioânia. Fueron realizadas: entrevista y evaluación de AVD y AIVD. Resultados: Participaron 95 ancianos, con predominio del grupo etáreo de 60 a 69 años (60\%), sexo femenino (60\%), estod civil casado (49,5\%), religión católica (54,7\%) ingreso menor o igual a 1 salario mínimo (45,3\%) y morada propia $(89,5 \%)$. Fueron identificados independencia máxima en las AVD $(57,9 \%)$ y compromiso en las AIVD (72,6\%). Conclusión: Los participantes presentaron, predominantemente, independencia para las actividades en el domicilio y dependencia para las actividades fuera de él.

Descriptores: Salud del anciano; Atención primaria de salud; Enfermería geriátrica; Programa salud de la familia.

\footnotetext{
${ }^{1}$ Enfermeiro

${ }^{2}$ Doutora em Enferma em aculdade de Enfermagem da Universidade ederal de oi s $O$ oi nia ( O), Brasil.
} 


\section{INTRODUÇÃO}

Diante do envelhecimento populacional, a meta no atendimento à saúde deixa de ser a de apenas prolongar a vida, mas, principalmente, a de manter a capacidade funcional do indivíduo, de forma que esse permaneça autônomo e independente pelo maior tempo possível. Para que isso ocorra, o sistema de saúde precisa garantir o acesso universal aos cuidados progressivos de saúde e as políticas públicas devem enfatizar a promoção de saúde e a prevenção de doenças. Além disso, o idoso deve ser avaliado de forma holística, com o objetivo principal de manutenção da capacidade funcional ${ }^{(1)}$.

Para mensurar a capacidade do idoso em relação à autonomia e à independência, existe um conjunto de dados clínicos, testes e escalas denominadas de Avaliação Funcional. Uma avaliação funcional simples deve conter avaliações: do equilíbrio e mobilidade, da função cognitiva, da capacidade para executar a atividade de vida diária (AVD) e as atividades Instrumentais de Vida Diária $(\mathrm{AIVD})^{(2)}$

As AVD são as tarefas que uma pessoa precisa realizar para cuidar de si, tais como: tomar banho, vestir-se, ir ao banheiro, andar, comer, passar da cama para a cadeira, mover-se na cama e ter continências urinária e fecal. As AIVD são as habilidades do idoso para administrar o ambiente em que vive e inclui as seguintes ações: preparar refeições, fazer tarefas domésticas, lavar roupas, manusear dinheiro, usar o telefone, tomar medicações, fazer compras e utilizar os meios de transporte ${ }^{(2)}$.

Diversos estudos têm projetado um grande crescimento da população idosa funcionalmente incapacitada, sendo que o número de pessoas idosas dependentes nas AVD dobrará na segunda ou terceira década deste século. Para aumentar as chances de um envelhecimento saudável, as políticas sociais e de saúde devem promover a habilidade funcional e construir um sistema adequado de suporte social para a população idosa ${ }^{(3)}$.

O envelhecimento é um processo multifatorial e ocorre de forma distinta, considerando as diferentes regiões geográficas. Nesse contexto, as habilidades para realização de AVD e AIVD podem apresentar-se de forma bastante diversificada.

O atual sistema de saúde focaliza a expansão da Saúde da Família. Para atender a população idosa é necessário que a equipe de saúde da família conheça o perfil sócioeconômico-demográfico, bem como a capacidade funcional da população idosa da sua região, para que possa planejar e implementar ações de promoção da saúde, prevenção e tratamento de agravos e reabilitação. Em Goiânia, informações nesse sentido são escassas e dispersas, dificultando o planejamento de ações de maior resolutividade coletiva.

Acreditamos que, o presente estudo poderá contribuir, direcionando o atendimento à população, criando subsídios para o ensino de gerontogeriatria e pesquisas futuras, em prol de um envelhecimento saudável.

\section{OBJETIVOS}

- Caracterizar o perfil dos idosos atendidos por uma equipe de PSF de Goiânia (GO) quanto a: sexo, idade, estado civil, religião, escolaridade, renda e moradia;

- Avaliar a capacidade desses idosos para desenvolver atividades de vida diária e atividades instrumentais de vida diária.

\section{MÉTODOS}

Trata-se de pesquisa descritiva realizada no Distrito Sanitário Leste do Município de Goiânia (GO), na sua mais recente área de implantação de PSF, na região, que é a equipe dois do Recanto das Minas Gerais.

Este estudo foi aprovado pelo Comitê de Ética em Pesquisa (COEP) da Universidade Federal de Goiás e pelo Gestor da área de abrangência onde foi realizada a pesquisa.

A população local de idosos totalizou 116 indivíduos com idade igual ou superior a 60 anos, dos quais 95 $(81,9 \%)$ foram incluídos na amostra. Todos foram informados sobre a natureza do estudo e ao concordarem em participar, assinaram o Termo de Consentimento Livre e Esclarecido, atendendo aos aspectos éticos de pesquisa com seres humanos ${ }^{(4)}$. O número de idosos que não aceitaram participar foram 16, e 6 não foram encontrados nos respectivos domicílios.

A coleta de dados foi realizada no domicílio do idoso, no ano de 2004. O protocolo incluiu itens de identificação: sexo, idade, estado civil, religião, escolaridade, renda e tipo de moradia. Além disso, foi utilizada a escala de Barthel ${ }^{(2)}$, para mensurar a capacidade em desenvolver as Atividades de Vida Diária (AVD), e a escala de Lawton ${ }^{(2)}$, para mensurar a capacidade em desenvolver as Atividades Instrumentais de Vida Diária (AIVD).

Para análise dos resultados foram utilizados procedimentos de estatística descritiva (freqüências simples e percentuais)

\section{RESULTADOS E DISCUSSÕES}

A faixa etária predominante foi de 60 a 69 anos (60\%), seguindo a dos 70 a 79 anos $(31,6 \%$ ) e a dos 80 a 89 anos $(8,4 \%)$. Considerando as duas primeiras faixas etárias, pode-se dizer que este perfil foi semelhante ao da população brasileira, em que o grupo de 60-69 anos soma $56,3 \%$ e o de $70-79$ anos totaliza $31,1 \% \%^{(5)}$. Em relação à faixa etária de 80-89 anos foi encontrado um percentual 
menor que o existente, nos idosos, na população brasileira, que representa $10,8 \%{ }^{(1)}$. Nessa área, não foram identificados idosos, com mais de 90 anos, o que existe na população brasileira, com uma freqüência de $1,8 \%{ }^{(5)}$. O perfil desta área geográfica apresenta características de uma ocupação recente.

Quanto ao sexo, 60\% eram mulheres e 40\% homens. Estes achados foram spróximos ao perfil do território nacional. De acordo com censo demográfico de 2000, no país, $55,1 \%$ da população idosa foi do sexo feminino e $44,9 \%$ do masculino ${ }^{(5)}$.

Em relação ao estado civil predominaram os casados (49,5\%) e viúvos (34,7\%). Os resultados do censo 2000 demonstraram que os idosos casados da população brasileira totalizavam $51,8 \%$ e os viúvos $28,5 \%{ }^{(5)}$.

Referente à religião 54,7\% eram católicos, 38,9\% evangélicos, 1,1\% espíritas e 5,3\% não pertenciam a nenhuma religião. Percebeu-se menor percentual de pessoas da religião católica em relação à população brasileira, em geral, $(77,8 \%)$ e maior número de evangélicos $(12,4 \%)^{(5)}$.

Quanto à escolaridade, mais da metade dos idosos pesquisados eram alfabetizados $(53,7 \%)$ e possuíam o ensino fundamental incompleto. Os idosos analfabetos somavam 46,3\%. O acesso à escola, para este grupo foi menor que o da população brasileira, em geral, em que os idosos alfabetizados representavam $64,8 \%$ e os analfabetos $35,2 \%{ }^{(5)}$. A escolaridade dos idosos foi bastante variada, tanto no Estado de Goiás como no Brasil e no mundo ${ }^{(6-7)}$. Considerando a população mundial e a própria população brasileira, geralmente nas áreas mais pobres e menos desenvolvidas, a escolaridade é menor. O quadro se repete, considerando as micro-regiões dentro do mesmo município. Mapear estas realidades auxilia no planejamento de estratégias de educação de adultos e de educação em saúde.

No grupo estudado foi identificado que 45,3\% tinham renda igual ou menor que um salário mínimo, 44,2\% de um a três salários e 10,5\% acima de três salários. A renda desses idosos era menor, em comparação a média nacional, em que 27,4\% recebiam até um salário mínimo, $14,4 \%$ de um até três salários e 58,2\% mais que três salários ${ }^{(5)}$.

A maioria dos idosos possuía moradia própria (89,5\%) e o restante $(10,5 \%)$ morava em casa alugada. Trata-se de uma região caracterizada por movimentos de invasão, com posterior regularização da situação, mediante interferência dos órgãos públicos.

Com relação à capacidade para realizar as AVD, foi identificado que $57,9 \%$ dos idosos eram independentes, $37,9 \%$ dependentes para a minoria das AVD e 4,2\% dependentes para a maioria destas atividades, perfazendo $42,1 \%$ os que apresentavam algum grau de dependência.

Estes dados foram semelhantes aos encontrados em área geográfica próxima a esta região de Goiânia ${ }^{(6)}$. Entretanto, no sudeste do Brasil o percentual encontrado de independência para AVD foi menor ${ }^{(8-10)}$, talvez pela maior longevidade dos idosos, daquela região.

O comprometimento na independência para realizar as AVD antes dos 70 anos, no grupo de idosos pesquisados, revela um envelhecimento mal sucedido, provavelmente devido às condições sociais e econômicas adversas, que influenciaram negativamente no estado de saúde.

Mesmo no envelhecimento saudável, a partir dos 80 anos se espera algum grau de comprometimento fisiológico na capacidade de realização das AVD. A intensidade e a freqüência deste comprometimento são muito variadas, dependendo das condições gerais de saúde, ao longo da vida, e do modo de vida das pessoas em cada contexto sócio-econômico-histórico-cultural. No estudo realizado na cidade de São Paulo, apenas 15\% dos idosos com idade superior a 80 anos não necessitavam de auxílio de outras pessoas, enquanto $28 \%$ eram incapazes de desenvolver pelo menos cinco atividades da vida diária ${ }^{(9)}$.

Em relação à dependência, conforme mostra a Tabela 1, os idosos precisavam de ajuda, principalmente para usar escadas (45\%), banhar-se $(15 \%)$, deambular $(10 \%)$, comer $(7,5 \%)$, vestir-se $(7,5 \%)$, passar da cama para a cadeira $(7,5 \%)$ e mover-se na cama $(5 \%)$.

As atividades para as quais alguns idosos eram totalmente dependentes incluíam: usar escadas $(10 \%)$; banhar-se $(7,5 \%)$, vestir-se $(7,5 \%)$, usar o vaso sanitário $(7,5 \%)$, passar da cama para cadeira $(5,0 \%)$, deambular $(2,5 \%)$, comer $(2,5 \%)$ e mover-se na cama $(2,5 \%)$.

Ainda com relação à dependência para realizar as AVD, pode ser observado nesta Tabela que $77,5 \%$ dos idosos apresentaram algum tipo de incontinência para as micções e $27,5 \%$ para as evacuações.

Essa ocorrência pode ser considerada alta em relação aos dados disponíveis na literatura ${ }^{(11-12)}$. Ao mesmo tempo, é preocupante, uma vez que a falta de controle dos esfíncteres pode levar ao isolamento social, alterações na auto-estima e auto-imagem, podendo influenciar também nas atividades instrumentais de vida diária.

A prevalência de dependência para a realização das AVD (42,1\%) foi semelhante a encontrada na região próxima cuja ocorrência foi de $40,9 \%$ de idosos ${ }^{(6)}$. No Estado de São Paulo esse comprometimento foi maior ${ }^{(8,}$ 10), provavelmente como já afirmamos anteriormente, pela idade mais avançada dos idosos, daquelas cidades.

A mobilidade articular permite a realização de atividades comuns da vida diária e, freqüentemente, encontra-se diminuída nos idosos sedentários, levando à diminuição de flexibilidade. Neste sentido, o comprometimento das AVD, pode ser evitado ou revertido se o idoso for orientado por um profissional 
Tabela 1: Distribuição de 40 idosos segundo a dependência para realizar AVD de acordo com a escala de Barthel. PSF. Equipe 2/ Recanto das Minas Gerais, Goiânia, 2004.

\begin{tabular}{llrr}
\hline Dependência para realizar as Atividades de Vida Diária* & $\mathbf{N}$ & $\mathbf{\%}$ \\
\hline Precisa de ajuda & para usar escadas & 18 & 45,0 \\
& para mover-se na cama & 2 & 5,0 \\
& para comer & 3 & 7,5 \\
& para se vestir & 3 & 7,5 \\
Totalmente ependente & para o banho & 6 & 15,0 \\
& para deambular & 4 & 10,0 \\
& para passar cama / cadeira & 3 & 7,5 \\
& para o banho & 3 & 7,5 \\
& para se vestir & 3 & 7,5 \\
Incontinência ocasional & para comer & 1 & 2,5 \\
& para deambular & 1 & 2,5 \\
Incontinência & para usar escadas & 4 & 10,0 \\
& para passar cama / cadeira & 2 & 5,0 \\
*Dos 40 idosos dependentes para a realização & par AVD, muitos são dependentes em mais de uma atividade
\end{tabular}

competente, e iniciar um programa de atividades físicas, com objetivo de melhorar a mobilidade articular e força muscular.

Com relação à capacidade de realizar as AIVD, foi identificado que $27,4 \%$ eram independentes, $68,4 \%$ apresentavam dependência parcial, sendo considerados limítrofes e 4,2\% apresentavam dependência total, perfazendo $72,6 \%$ de idosos com dificuldades para administrar o ambiente em que viviam e lidarem com a vida social de forma autônoma. Esse grau de dependência para as AIVD foi bem maior do que o identificado em área geográfica próxima ${ }^{(6)}$ na qual foi observado que $58,0 \%$ dos idosos eram dependentes em uma ou mais atividades.

As três AIVD nas quais os idosos tinham mais dependência, necessitando de ajuda, foram: manuseio de dinheiro $(73,9 \%)$, uso de meios de transporte $(72,5 \%)$ e trabalho doméstico $(40,6 \%)$. As três atividades em que os idosos tinham maior dependência total incluíam: lavar qualquer peça de roupa $(30,4 \%)$, realizar trabalho doméstico $(26,1 \%)$ e utilizar o telefone $(20,3 \%)$, conforme pode ser observado na Tabela 2.

A dependência funcional pode levar à perda de autonomia. $\mathrm{Na}$ medida em que um idoso demonstra algum grau de dependência, para administrar seus recursos financeiros ou adquirir alimentos e bens de consumo básico, pode ter sua autonomia prejudicada. Às vezes, torna-se necessário a ajuda de outras pessoas para gerir seus bens financeiros, fazer suas compras e realizar os afazeres domésticos ${ }^{(13)}$.

A incapacidade na realização de uma dessas atividades instrumentais de vida diária, além de prejudicar a vida social do idoso, potencialmente implica em transtornos para ele e sua família, a qual, dependendo da atividade, terá que mobilizar maior tempo disponível, energia e recursos financeiros para suprir as demandas existentes.

Algumas incapacidades se associam à falta de escolaridade como: manusear dinheiro, tomar medicação, usar o telefone, fazer compras e utilizar meios de transporte, segundo relataram os idosos entrevistados. Tais incapacidades comprometem sobremaneira a socialização dos mesmos, diminuindo sua autonomia. É preciso tomar providências em relação a esta situação, proporcionando adaptações que favoreçam condições de acesso aos conhecimentos e recursos, que poderão torná-los autônomos e independentes.

Em qualquer contexto de atendimento a idosos, seja no PSF ou em um hospital, é preciso que os profissionais de saúde, e em especial da enfermagem, estejam atentos para esta avaliação, na perspectiva de identificar problemas dos idosos para a sistematização da assistência de enfermagem $^{(14)}$.

\section{CONCLUSÕES}

O perfil do grupo de idosos da área pesquisada revelou uma população predominantemente feminina, casada, na faixa etária dos 60 a 69 anos, católica, 
Tabela 2: Distribuição de 69 idosos segundo a dependência para realizar as Atividades Instrumentais de Vida Diária de acordo com escala de Lawyon. Equipe 2/Recanto das Minas Gerais, Goiânia, 2004.

\begin{tabular}{|c|c|c|}
\hline Dependência para realizar as AIVD & $\mathbf{N}$ & $\%$ \\
\hline \multicolumn{3}{|l|}{ Preparar as refeições } \\
\hline incapaz de preparar as refeições & 14 & 20,3 \\
\hline necessita de ajuda para preparar as refeições & 7 & 10,1 \\
\hline \multicolumn{3}{|l|}{ Trabalbo doméstico } \\
\hline capaz de realizar apenas trabalho doméstico leve & 28 & 40,6 \\
\hline incapaz de realizar qualquer trabalho doméstico & 18 & 26,1 \\
\hline \multicolumn{3}{|l|}{ Lavar roupa } \\
\hline capaz de lavar peças pequenas & 24 & 34,8 \\
\hline incapaz de lavar qualquer peça & 21 & 30,4 \\
\hline \multicolumn{3}{|l|}{ Tomar medicação } \\
\hline necessita de lembretes para tomar a medicação & 20 & 30,0 \\
\hline incapaz de tomar medicação & 9 & 13,0 \\
\hline \multicolumn{3}{|l|}{ Utilizar o telefone } \\
\hline necessita de ajuda ou de aparelho especial para discar & 27 & 39,1 \\
\hline incapaz de usar o telefone & 14 & 20,3 \\
\hline \multicolumn{3}{|l|}{ Manuseio de dinbeiro } \\
\hline necessita de ajuda com cheques e pagamentos de contas & 51 & 73,9 \\
\hline incapaz de lidar com dinheiro & 10 & 14,5 \\
\hline \multicolumn{3}{|l|}{ Compras } \\
\hline necessita de supervisão para fazer compras & 27 & 39,1 \\
\hline incapaz de realizar as compras, mesmo com supervisão & 13 & 18,8 \\
\hline \multicolumn{3}{|l|}{ Uso de meios de transporte } \\
\hline necessita de ajuda quando viaja de ônibus, trens, metrôs e táxi & 50 & 72,5 \\
\hline incapaz de utilizar qualquer meio de transporte & 10 & 14,5 \\
\hline
\end{tabular}

alfabetizada, com baixa renda mensal e residente em moradia própria.

Foi observado que $57,9 \%$ dos idosos eram independentes para as AVD. As áreas em que estas atividades estão comprometidas, parcialmente, em ordem decrescente de freqüência foram: controle dos esfíncteres urinário e anal, usar escadas, banhar-se, deambular, comer, vestir-se, passar da cama para cadeira e mover-se no leito. As atividades em que foi identificada dependência total incluem: usar escada, banhar-se, vestir-se, usar o vaso sanitário, passar da cama para a cadeira, comer, deambular e mover-se na cama.

Com relação ao desenvolvimento de atividades instrumentais de vida diária a maioria $(72,6 \%)$ era dependente parcial ou totalmente, precisando de ajuda, principalmente para: o manuseio de dinheiro, a utilização de meios de transporte, realizar compras, realizar trabalho doméstico e lavar roupa. Eram totalmente dependentes para: lavar roupas, usar telefone, preparar refeições e realizar compras.

Tendo em vista que a capacidade funcional do ser humano declina com a idade, é necessário planejar estratégias que melhorem o estilo de vida dos idosos dessa região, principalmente em relação a programas que proporcionem: promoção e melhoria da força muscular e de articulação; promoção, tratamento e reabilitação da capacidade funcional dos esfíncteres urinário e intestinal; integração social dentro e fora do contexto familiar; construção de um sistema adequado de suporte ao idoso; educação permanente ao longo da vida e valorização do processo de envelhecimento individual e populacional.

Tais ações possibilitariam minimizar a dependência nas AVD e as AIVD, proporcionado assim, um envelhecimento com autonomia e independência às pessoas idosas.

Acreditamos que estudos dessa natureza sejam necessários nas micro-regiões, em cada área de abrangência de equipes de PSF, para que se possa obter um panorama mais próximo das micro realidades de saúde da população, objetivando assim, bases mais seguras e concretas, para o atendimento integral à saúde.

\section{REFERÊNCIAS}

1. Costa EFA, Porto CC, Soares AT. Envelhecimento populacional brasileiro e o aprendizado de geriatria e gerontologia. Rev UFGO. 2003; 5(2): 7-10.

2. Costa EFA, Porto CC, Almeida JC, Cipullo JP, Martin JFV. Semiologia do idoso. In: Porto CC. Semiologia médica. 4a ed. Rio de Janeiro: Guanabara Koogan; 2001. p.165-197.

3. Paschoal SMP. Autonomia e Independência. In: Papaléo Netto M. Gerontologia: a velhice e o envelhecimento em visão globalizada. São Paulo: Atheneu; 2002. p. 313-323.

4. Conselho Nacional de Saúde. Comitê Nacional de 
Ética em pesquisa em Seres Humanos. Resolução 196, de 10 de outubro de 1996: Diretrizes e normas regulamentadoras de pesquisa envolvendo seres humanos. Diário Oficial da União Brasília (DF); 1996 Out 16, n. 201, Seção 1:21082.

5. Instituto Brasileiro de Geografia e Estatística. IBGE. Censo Demográfico 2000. Perfil dos idosos responsáveis pelos domićlilios no Brasil 2000. Tabela 1 - População residente, total e de 60 anos ou mais de idade, por sexo e grupos de idade, segundo as Grandes Regiões e Unidades da Federação - 1991/ 2000. Brasília (DF);2000. Disponível em: URL: $<$ http://www.ibge.com.br/home/estatistica/ populacao/perfilidoso/tabela1 6.shtm $>$. Acessado em: 10 mar 2006.

6. Nakatani AYK, Costa EFA, Teles SA, Silva LB, Rêgo MP, Silva e Souza AC, et al. Perfil sócio-demográfico e avaliação funcional de idosos atendidos por uma equipe de saúde da família na periferia de Goiânia, Goiás. Rev Soc Bras Clin Méd. 2003; 1(5): 131-6.

7. Sousa L, Galante H, Figueiredo D. Qualidade de vida e bem-estar dos idosos: um estudo exploratório na população portuguesa. Rev Saúde Pública. 2003; 37(3): 364-71.

8. Ramos LR, Rosa TEC, Oliveira ZMA, Medina MCG, Santos FRG. Perfil do idoso em área metropolitana na região sudeste do Brasil: resultados de inquérito domiciliar. Rev Saúde Pública. 1993; 27( 2): 87-94.

9. Ramos LR, Saad PM. Morbidade na população idosa. In: Fundação SEADE . O idoso na Grande São Paulo. São Paulo: SEADE; 1990. p. 161- 72. (Coleção Realidade Paulista).

10. 10 Feliciano AB, Moraes SA, Freitas ICM. O perfil do idoso de baixa renda no Município de São Carlos, São Paulo, Brasil: um estudo epidemiológico. Cad Saúde Pública. 2004; 20 (6):1575-85.

11. Araújo SEA, Habr-Gama A. Incontinência fecal. In: Brasil. Instituto para o Desenvolvimento da Saúde; Universidade de São Paulo; Ministério da Saúde. Manual de Condutas Médicas. Brasília: Ministério da Saúde; 2002. 461p. p.388-390.

12. Arap MA, Gomes CM. Incontinência urinária. In: Brasil. Instituto para o Desenvolvimento da Saúde; Universidade de São Paulo; Ministério da Saúde. Manual de Condutas Médicas. Brasília: Ministério da Saúde; 2002. p.391.

13. Brasil. Ministério da Saúde. Atenção à Saúde do Idoso - Instabilidade postural e queda. Brasília: Ministério da Saúde. Secretaria de Políticas de Saúde. Departamento de Atenção Básica;1999. 40p. [Organizado por: Milton Menezes da Costa Neto ].

14. Paula JC, Cintra FA. A relevância do exame físico do idoso para assistência hospitalar. Acta Paul Enferm. 2005; 18(3):301-6. 